\title{
Um estudo sobre a provisão de qualidade de serviço nas redes de acesso WiMAX
}

\author{
Wyllian Bezerra da Silva, Anauto Lucas Maciel Mendes, Paulo Roberto Guardieiro
}

\begin{abstract}
Resumo - Nos últimos anos, em todo o mundo, ocorreu um aumento na disponibilidade de acesso em banda larga (p. ex., via cable modem, ADSL). Todavia, em áreas rurais e suburbanas o custo para a disponibilização deste recurso tem limitado o seu crescimento. Nestes casos, a tecnologia BWA (Broadband Wireless Access) das redes de acesso WiMAX (IEEE 802.16) representa uma solução promissora, além de atender aos diferenciados requisitos de serviços das aplicações multimídia. Neste trabalho, apresenta-se um estudo sobre a provisão de qualidade de serviço (QoS) usando modelagem e simulação.
\end{abstract}

\section{Palavras-Chave - IEEE 802.16, WiMAX, BWA, QoS.}

Abstract - In the last years, in the whole world, an increase in the availability of access in broadband occurred (e.g., cable modem, ADSL). However, in rural and suburban areas the cost to become available this resource has limited its growth. In these cases, technology BWA (Broadband Wireless Access) of the WiMAX access networks (IEEE 802.16) represents a promising solution, besides taking care of to the differentiated requirements of services of the applications multimedia. In this work, a study is presented on the provision of quality of service (QoS) using modeling and simulation.

Keywords - IEEE 802.16, WiMAX, BWA, QoS.

\section{INTRODUÇÃO}

Nos últimos anos, em todo mundo, ocorreu um aumento na disponibilidade de redes de acesso em banda larga baseadas em cable modem, ADSL e enlaces de fibras ópticas. Este fato proporcionou um elevado crescimento no número das aplicações multimídia, tais como VoIP, videoconferência, vídeo sob demanda etc. Todavia, em áreas rurais e suburbanas, o custo para a disponibilização deste recurso de acesso tem limitado o seu crescimento. Nestes casos, a tecnologia BWA (Broadband Wireless Access) das redes de acesso WiMAX (IEEE 802.16) [1] representa uma solução promissora. O termo WiMAX significa Worldwide Interoperability for Microwave Access. Esta tecnologia estabelece uma interface área para redes metropolitanas sem fio (Wireless MAN). Oficialmente, este padrão é denominado de Interface Aérea para Sistemas Fixos de Acesso Sem Fio de Banda Larga (Air Interface for Fixed Broadband Wireless Systems) e define uma rede metropolitana sem fio, provendo acesso por meio de antenas externas que se comunicam com uma estação base (Base Station - BS). O padrão IEEE 802.16 especifica dois

Wyllian Bezerra da Silva, Anauto Lucas Maciel Mendes, Paulo Roberto Guardieiro, Laboratório de Redes de Computadores, Faculdade de Engenharia Elétrica, Universidade Federal de Uberlândia, Uberlândia, Minas Gerais, Brasil. E-mails: wyllian@mestrado.ufu.br, alucas@mestrado.ufu.br, prguardieiro@ufu.br. modos de compartilhamento do meio sem fio: pontomultiponto (Point-to-Multipoint - PMP) e mesh (opcional).

O grande desafio das redes BWA é a provisão de qualidade de serviço (Quality of Service - QoS) às aplicações multimídia com seus diferentes requisitos de serviço. Isto se deve ao fato de que estas redes são sem fio, o que dificulta a provisão de QoS em relação às redes cabeadas, uma vez que os enlaces sem fio são altamente variáveis e imprevisíveis. Para superar estes problemas, o gerenciamento da QoS nas redes sem fio é feito na camada MAC (medium access control). O padrão IEEE 802.16 incorpora na camada MAC a capacidade de gerenciamento do tráfego gerado pelas aplicações multimídia com seus diferentes requisitos de QoS.

Embora a literatura pesquisada indique a existência de trabalhos de análise da camada MAC do IEEE 802.16, algumas questões ainda não foram abordadas. Em [4] é apresentada uma análise do mecanismo para obtenção de QoS no padrão IEEE 802.16, todavia, considera-se apenas o escalonamento DRR (Deficit Round-Robin). Em [5] descreve-se uma breve análise da vazão e atraso na camada MAC considerando o escalonamento WRR (Weighted Round-Robin). Assuntos relacionados ao escalonamento e/ou policiamento não são obrigatórios no padrão IEEE 802.16, isto possibilita liberdade quanto à sua implementação. Esta questão é discutida em [6] que propõe uma nova estratégia de escalonamento, utilizando diversos métodos de policiamento, conforme o tipo de classe de serviço.

Neste artigo apresenta-se uma revisão e análise do mecanismo para provisão de QoS na camada MAC definido pelo padrão IEEE 802.16. Neste estudo baseado em modelagem e simulação, considera-se uma rede de acesso PMP submetida aos tráfegos UGS, rtPS, ertPS, nrtPS e BE.

Este artigo é organizado da seguinte forma: na Seção II, descreve-se o mecanismo para provisão de QoS na camada MAC do padrão IEEE 802.16. Na Seção III apresenta-se o estudo baseado em modelagem e simulação desenvolvido neste trabalho, descrevendo-se o ambiente de rede e os parâmetros de simulação considerados. Os resultados de simulação e a análise dos mesmos são apresentados na Seção IV. Finalmente, na Seção V apresentam-se as conclusões deste trabalho.

\section{QOS EM REDES WIMAX}

A QoS em redes WiMAX, padrão IEEE 802.16, está associada a conceitos como: classificação, escalonamento por fluxos de serviço e estabelecimento de serviço. O mecanismo mais importante na provisão de QoS está relacionado com a transferência de pacotes da interface MAC, através de um fluxo de serviço identificado de forma única, por meio de um identificador de conexão CID (Connection IDentifier). O fluxo de serviço é um fluxo unidirecional de pacotes ao qual está associado um nível de QoS específico [6]. A SS e a BS garantem a qualidade de 
serviço de acordo com o conjunto de parâmetros de QoS definidos para o fluxo.

A QoS é obtida via fluxo de serviços, o qual é responsável pela transmissão unidirecional dos pacotes provenientes da SS (uplink) ou da BS (downlink). Este fluxo de pacotes é caracterizado por um conjunto de parâmetros de QoS, tais como: latência, retardo e garantias de vazão. Com o propósito de padronizar a operação entre a SS e a BS, os fluxos MAC-PDU são classificados e enviados às SSs, mediante a verificação dos parâmetros pertencentes à conexão MAC, conforme mostrado na Figura 1. Estes parâmetros incluem detalhes de como a SS solicita mini-slots para uplink e o comportamento esperado do escalonador de uplink na BS.

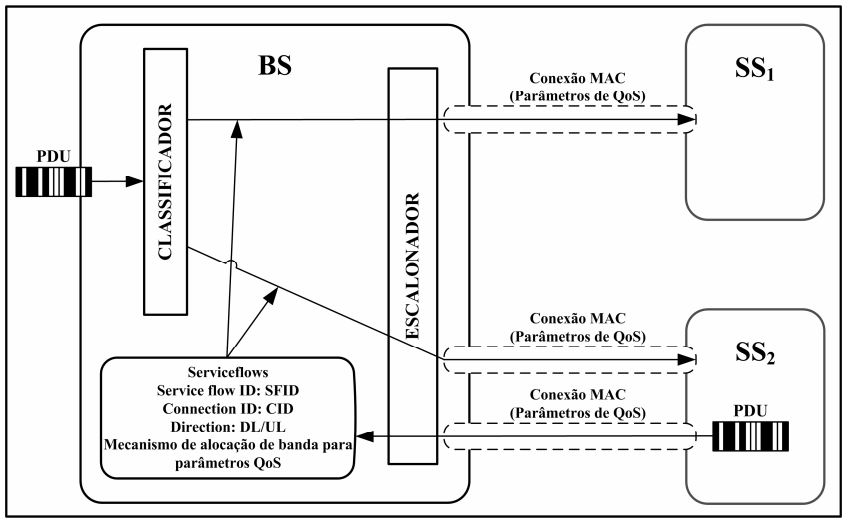

Figura 1 - Estabelecimento de QoS entre BS e SS.

A todos os fluxos de serviços existentes atribui-se um identificador de fluxo de serviço (Service Flow Identifier SFID), o qual serve como o principal identificador na SS e na BS para cada fluxo. Um fluxo de serviço tem no mínimo um SFID e uma direção associada. No CID, o mapeamento para um SFID somente é realizado quando a conexão tem seu fluxo de serviço admitido. Um conjunto de parâmetros de QoS (ProvisionedQoSParamSet) é fornecido por mecanismos externos aos definidos no padrão 802.16, como, por exemplo, pelo sistema de gerenciamento de rede. Os parâmetros de QoS são definidos pelo AdmittedQoSParamSet nos quais a BS (e possivelmente a SS) reserva recursos. O principal recurso reservado é a largura de banda, mas outros recursos também podem ser reservados (por exemplo, espaço em buffer) para viabilizar a ativação do fluxo. O ActiveQoSParamSet especifica um conjunto de parâmetros de QoS que define o tipo de serviço provido para o fluxo de serviço. Somente um fluxo de serviço ativo pode encaminhar pacotes.

O relacionamento entre os conjuntos de parâmetros de QoS está ilustrado na Figura 2 e na Figura 3. O ActiveQoSParamSet é sempre um subconjunto do AdmittedQoSParamSet, que é sempre um subconjunto do “envelope” de autorização. No modelo de autorização provisionado, esse envelope é determinado pelo ProvisionedQoSParamSet. Já no modelo de autorização dinâmico, esse envelope é determinado pelo módulo de autorização (rotulado como AuthorizedQoSParamSet).

Existem três tipos de fluxos de serviço: provisionado, admitido e ativo. O primeiro provisiona o sistema de gerenciamento de rede. Os conjuntos de parâmetros AdmittedQoSParamSet e ActiveQoSParamSet para esse tipo de fluxo são nulos. No fluxo admitido, os recursos são reservados pela BS para o seu conjunto de parâmetros AdmittedQoSParamSet, mas esses parâmetros não estão ativos (ActiveQoSParamSet é nulo). Os fluxos de serviço admitidos podem ter sido provisionados ou sinalizados por algum outro mecanismo. Já o fluxo ativo apresenta recursos comprometidos pela BS para o seu conjunto de parâmetros ActiveQoSParamSet. O conjunto de parâmetros ActiveQoSParamSet desse fluxo não é nulo.

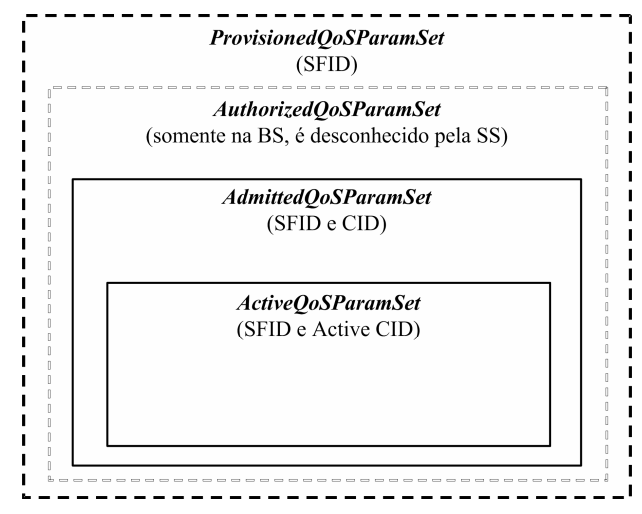

Figura 2: Modelo de autorização provisionado [1]

AuthorizedQoSParamSet $=$ ProvisionedQoSParamSet (SFID)

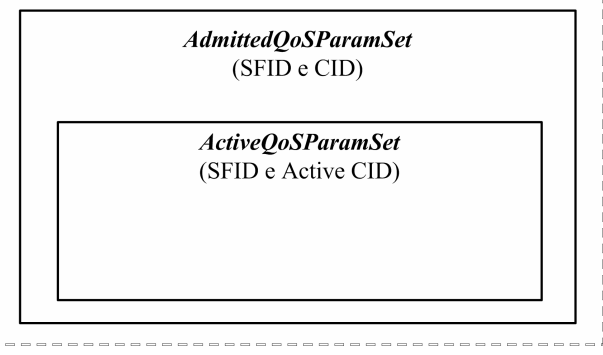

Figura 3: Modelo de autorização dinâmico [1].

O mecanismo principal para a provisão de QoS no padrão IEEE 802.16 é o mecanismo de classificação, conforme mostrado na Figura 4.

\section{Estação do Assinante (SS)}

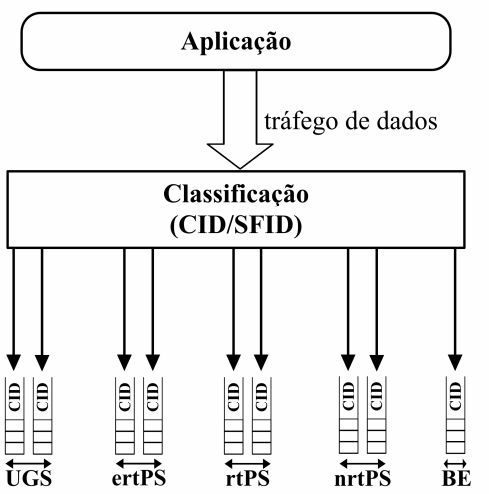

Figura 4: Provisão de QoS definida no padrão IEEE 802.16.

Cada aplicação deve primeiramente registrar-se na rede. A rede irá associar à aplicação a um fluxo de serviço através da atribuição de um SFID. Todos os pacotes devem ser rotulados com o SFID atribuído de modo que a rede possa prover a QoS apropriada. Quando a aplicação enviar pacotes de dados, ela solicitará o estabelecimento de uma conexão com a rede e receberá um CID. Dessa forma, os pacotes de dados do padrão IEEE 802.16 incluem identificadores por fluxo e por conexão.

$\mathrm{O}$ conjunto de ferramentas que oferece suporte à provisão de QoS para os tráfegos uplink e downlink inclui 
funções para configuração e registro dos fluxos de serviço, sinalização para estabelecimento dinâmico de QoS (baseado nos fluxos de serviço e nos parâmetros de tráfego), escalonamento e parâmetros de tráfego para fluxos uplink e downlink e agrupamento de propriedades do fluxo de serviço em classes de serviço (para agregar requisições).

O padrão IEEE 802.16 define cinco classes de tráfego: UGS (Unsolicited Grant Service), destinado ao tráfego com taxa constante de bits (Constant Bit Rate - CBR), para o tráfego em tempo real; rtPS (real-time Polling Service), serviço em tempo real com taxa variável de bits (Variable Bit Rate - VBR), também para o tráfego em tempo real, como vídeo MPEG (Motion Picture Expert Group); nrtPS (non real-time Polling Service), com VBR, porém, não é um serviço em tempo real, cujas aplicações são tolerantes a atrasos; BE (Best Effort), serviço destinado a tráfego cuja taxa é variável, sendo próprio para aplicações elásticas. Estas classes pertencem ao padrão IEEE 802.16-2004. Além delas, há um serviço adicional pertencente ao padrão IEEE 802.16c: ertPS (extendend real-time Polling Service), para aplicações em tempo real, como VoIP com supressão de silêncio.

\section{MODELAGEM E SIMULAÇÃO}

A utilização do recurso modelagem e simulação na área de redes de computadores é de fundamental importância, uma vez que permite a avaliação de parâmetros essenciais no seu estudo, tais como os parâmetros de qualidade de serviço. Para tanto, as análises aqui apresentadas foram realizadas a partir de resultados de simulação obtidos com o simulador Network Simulator 2 (ns-2) [2], ao qual foi agregado o módulo WiMAX descrito em [3]. Este módulo está sendo desenvolvido pelo NDSL (Networks \& Distributed Systems Laboratory). Em sua fase atual de desenvolvimento, ele não atende aos requisitos deste trabalho e sofreu aprimoramentos para adequá-lo ao presente estudo.

A Figura 5 ilustra a relação entre o módulo WiMAX e os demais módulos do ns-2. No topo desta figura está o Agente Gerador de Tráfego (Traffic Generating Agent TGA), seguido da camada de enlace (Link Layer - LL), da interface de fila (Interface Queue - IFQ), da camada de controle de acesso ao meio (MAC) e da camada física (channel).

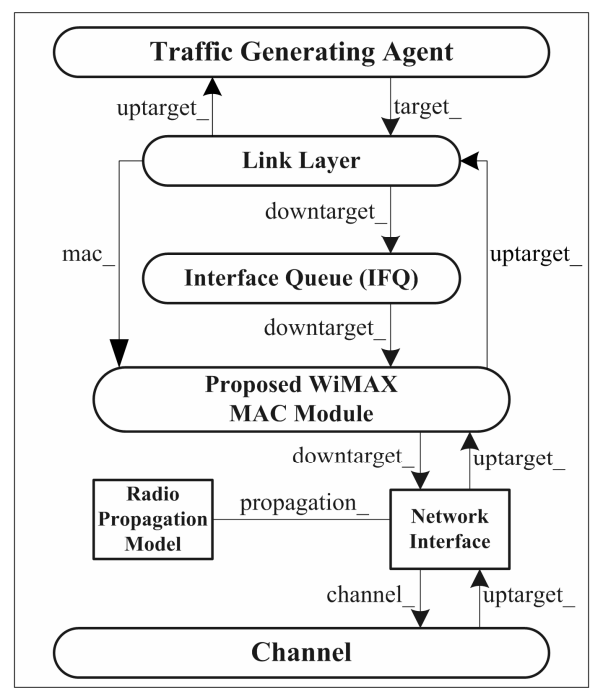

Figura 5: Relação entre os módulos WiMAX e o ns-2.
O TGA foi utilizado para modelar as camadas superiores, sendo que na camada de aplicação considera-se o tráfego gerado pelas aplicações Web, transferência de arquivos, VoIP e fluxo contínuo (streaming) de vídeo.

Para fins de análise do padrão IEEE 802.16 em termos da provisão de QoS, considerou-se dois cenários ad-hoc com diferentes condições de tráfego. No cenário 1, a BS está equidistantemente separada das cinco SSs aleatoriamente posicionadas ao seu redor, conforme mostrado na Figura 6.



Figura 6: Cenário 1 - Topologia da rede de acesso WiMAX.

No segundo cenário, além das considerações anteriores, as SSs estão submetidas a diferentes tipos de tráfego gerados por cinco aplicações distintas, conforme ilustrado na Figura 7.

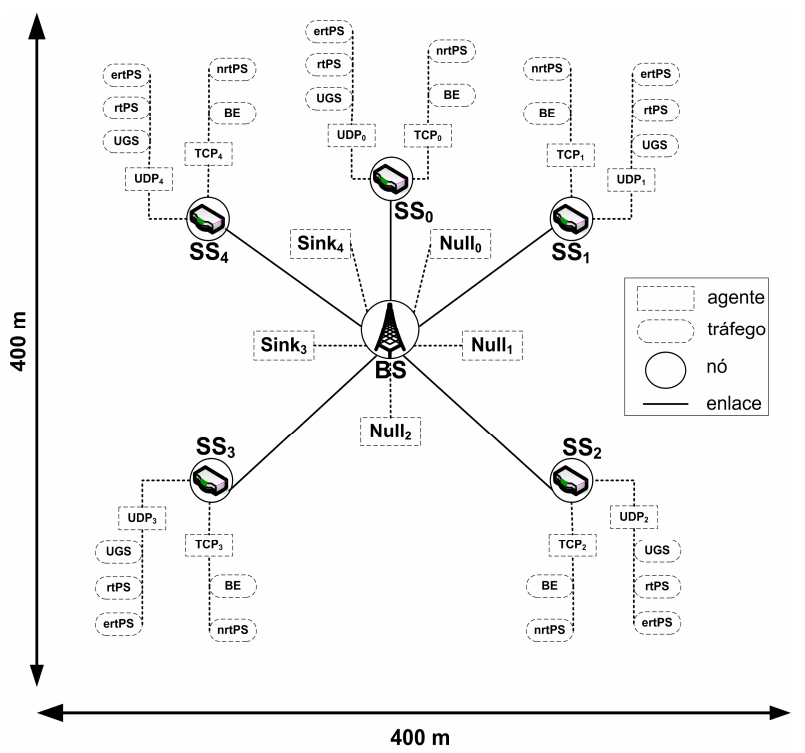

Figura 7: Cenário 2 - Topologia da rede de acesso WiMAX.

Para ambos os cenários, o tráfego gerado pelas aplicações concorrentes está caracterizado na Tabela 1. Considera-se um tempo de simulação de 60 segundos e ambientes de rede limitados a uma área de $400 \mathrm{~m}^{2}$. A antena é omnidirecional e o modelo de propagação utilizado é o de dois raios. A operação é no modo PMP, o que significa que a BS pode atender a múltiplas SSs concorrentemente. 
O tipo de gerenciamento de fila adotado na simulação é o DropTail, o método policiamento é o WRR (Weighted Round-Robin) cujas classes tem a seguinte ordem de prioridade: UGS $>$ rtPS $>$ ertPS $>$ nrtPS $>$ BE, com tamanho máximo de 50 pacotes presentes na fila. $\mathrm{O}$ protocolo de roteamento utilizado é o DSDV (Destination Sequenced Distance Vector) que cria uma tabela de rotas em cada nó, com o endereço do nó de destino e o número de saltos (hops), bem como o número de seqüência $\mathrm{SN}$ (Sequence Number).

A multiplexação OFDMA (Orthogonal Frequency Divison Multiple Access) foi considerada neste estudo. Outros parâmetros utilizados na simulação nas camadas MAC e física estão descritos nas Tabelas 2 e 3.

\section{APRESENTAÇÃO E ANÁLISE DE RESULTADOS}

Nesta seção são apresentados e analisados os resultados de simulação obtidos neste trabalho.

Inúmeras dificuldades foram encontradas na utilização do módulo WiMAX descrito em [3], para as quais algumas soluções foram encontradas, dentre elas, a inserção de novos agentes TCP e UDP, disponíveis em [8] e [9], respectivamente. Estes permitem coletar informações durante a simulação e gravar os dados diretamente em um arquivo. Através de programação em AWK [10], foi possível manipular os dados arquivados.

As análises foram feitas sobre valores médios. Dados estatísticos para os parâmetros do primeiro cenário estão na Tabela 4, com seus respectivos valores de desvios-padrão, médias e intervalos de confiança, ambos ao nível de confiança de $95 \%$.

Após um tratamento adequado dos dados, utilizou-se a ferramenta de plotagem gráfica GNUPLOT [11] para geração dos gráficos de vazão (throughput), atraso médio fim-a-fim, jitter e perda de pacotes.

A Figura 8 mostra os resultados obtidos para a vazão para o tráfego UGS, ertPS e rtPS considerando-se ambos os cenários e aplicações baseadas no UDP. Observa-se que a vazão para o tráfego rtPS se manteve constante, próximo a 1 Mbps, independentemente do cenário de tráfego. A vazão para o tráfego UGS e para o tráfego ertPS mostrou-se constante e igual a $64 \mathrm{kbps}$ e $58 \mathrm{kbps}$, respectivamente, em ambos os cenários.

A Figura 9 ilustra o comportamento da vazão, utilizando-se o TCP NewReno para as classes nrtPS e BE. A classe de tráfego nrtPS tem valores de vazão abaixo de 100 kbps no primeiro cenário, já no segundo cenário tem um ligeiro aumento, estabilizando-se acima de 150 kbps. A classe de tráfego BE produziu no primeiro cenário um nível de vazão abaixo de $15 \mathrm{kbps}$ e se manteve constante. Contudo, no segundo cenário decresce de 1 Mbps, aproximadamente e estabiliza-se próximo a $600 \mathrm{kbps}$. Tal comportamento resulta do fato de que esta classe aproveitou as sobras de recursos disponíveis neste cenário, pois as outras classes apresentam comportamentos menos suscetíveis a variações, corroborando para que esta classe tenha alguns níveis de QoS melhores do que a classe nrtPS.

A Figura 10 mostra os resultados de simulação para o atraso médio no tráfego baseado em UDP. Observa-se que nos dois cenários os comportamentos são semelhantes. O atraso médio para a categoria UGS apresentou-se constante e abaixo de $0,3 \mathrm{~ms}$. Na classe de tráfego rtPS verificou-se que o atraso médio se manteve constante e abaixo de $30 \mathrm{~ms}$.
A classe ertPS também apresentou valores de atrasos médios constantes próximos a $20 \mathrm{~ms}$.

Na Figura 11, o atraso médio para a classe BE e nrtPS, referente ao primeiro cenário, ficou estabilizado abaixo de 700 ms e 300 ms, respectivamente. Todavia, no segundo cenário, a classe nrtPS apresentou atraso médio mais elevado, próximo a $500 \mathrm{~ms}$ e a classe BE com valores abaixo de $5 \mathrm{~ms}$. Conforme descrito anteriormente, isto se deve ao comportamento da classe BE que aproveitou as sobras de recursos disponíveis no segundo cenário.

Os resultados de jitter para o tráfego nrtPS e BE estão representados na Figura 12 com valores reduzidos no segundo cenário e mais expressivos no primeiro cenário. A Figura 13 apresenta os resultados de jitter para as classes UGS, ertPS e rtPS com valores coincidentes nos dois cenários: 0,02 ms, $12 \mathrm{~ms}$ e $16 \mathrm{~ms}$, respectivamente. Estes resultados são atribuídos ao tipo e comportamento das fontes de tráfego ao longo do tempo de simulação.

\section{CONCLUSÕES}

Neste artigo foi realizado um estudo baseado em modelagem e simulação a respeito da provisão da qualidade de serviço nas redes de acesso WiMAX (IEEE 802.16). Os resultados obtidos para a vazão, atraso médio e jitter permitiram concluir que estas redes de acesso suportam condições variadas de tráfego, atendendo aos requisitos de QoS das aplicações dentro de certos limites de carga de tráfego.

Na seqüência deste trabalho pretende-se propor uma nova estratégia de escalonamento otimizada para o IEEE 802.16, que possibilite às classes com menores privilégios maior acesso à largura de banda disponível.

\section{REFERÊNCIAS}

[1] IEEE 802.16 Working Group. IEEE Standard for Local and Metropolitan Area Networks - Part. 16: Air Interface for Fixed Broadband Wireless Access Systems. IEEE Std. 802.16-2004.

[2] ns-2. The Network Simulator. Disponível em: <http://www.isi.edu/nsnam/ns/>. Acesso em 2 de abril de 2007.

[3] Module NDSL. Module WiMAX, versão 2.03. Netowrks \& Distributed Systems Laboratory (NDSL). Disponível em: $<$ http://ndsl.csie.cgu.edu.tw/wimax_ns2.php>. Acesso em 2 de abril de 2007.

[4] C.Cicconetti, L. Lenzini, E. Mingozzi and C. Eklund, "Quality of service support in IEEE 802.16 networks”, IEEE Network, 20 (2). 50-55. March/April 2006.

[5] F. C.Tsai, J. Chen, C. W. Chang, W. J. Lien, C. H. Hung, J. H. Sum, "The Design and Implementation of WiMAX Module for ns-2 Simulator", Proc. ACM VALEUTOOLS 2006, Pisa, Italy, October 10, 2006.

[6] M. Settembr, M. Puleri, S. Garritano, P. Testa, R. Albanese, M. Mancini, V. Lo Curto, "Performance Analysis of an Efficient PacketBased IEEE 802.16 MAC Supporting Adaptive Modulation and Coding”, Computer Networks, 2006. ISCN, June 2006, pp 11-16.

[7] Cisco Systems. Quality of Service (QoS). Disponível em <http://www.cisco.com/univercd/cc/td/doc/cisintwk/ito_doc/qos.htm>. Acesso em 20 de abril de 2007.

[8] CHIH-HENG, K. An example to measure the throughput of TCP-based application over wire-cum-wireless environment. Disponível em:

$<$ http://140.116.72.80/ smallko/ns2/measure_tcp.htm>. Acesso em 2 de abril de 2007.

[9] CHIH-HENG, K. How to measure packet loss rate, jitter, and end-toend delay for UDP-based applications. Disponível em: $<$ http://140.116.72.80/ smallko/ns2/tool_en.htm $>$. Acesso em 2 de abril de 2007.

[10] AHO, A. V.; KERNIGHAN, B. W.; WEINBERGER, P. J. The Programming Language AWK. Addison-Wesley, 1998. 
[11] WILLIAMS, T; HECKING, L.; BROEKER, H. -B. GNUPLOT, versão 4.2 (revisada em 3 de março de 2007), baseado na versão original

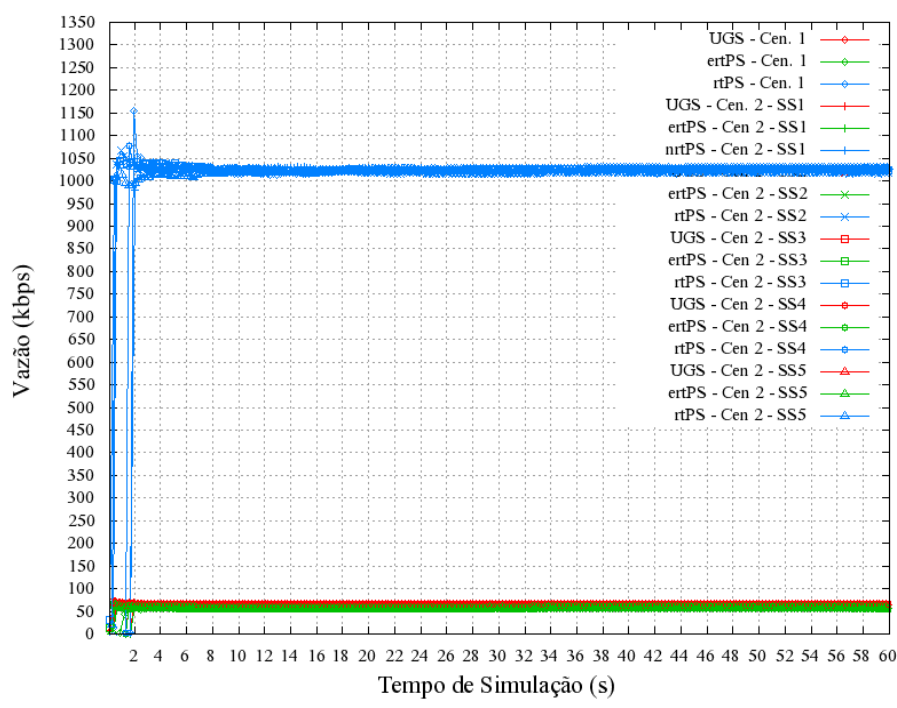

Figura 8: Vazão para o tráfego UDP: rtPS, ertPS e UGS.

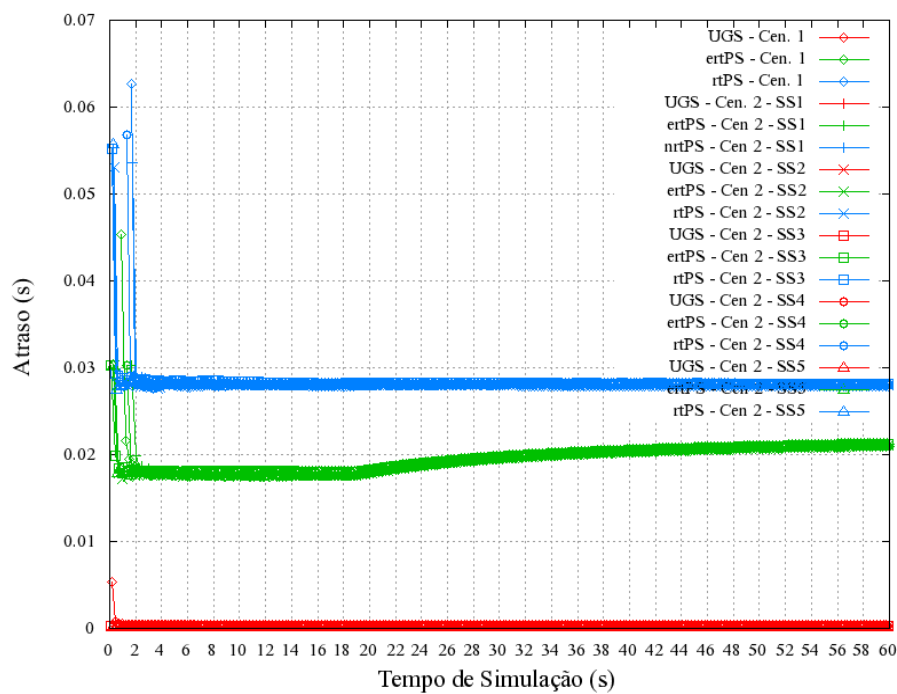

Figura 10: Atraso médio para tráfegos em UDP: UGS, ertPS e rtPS.

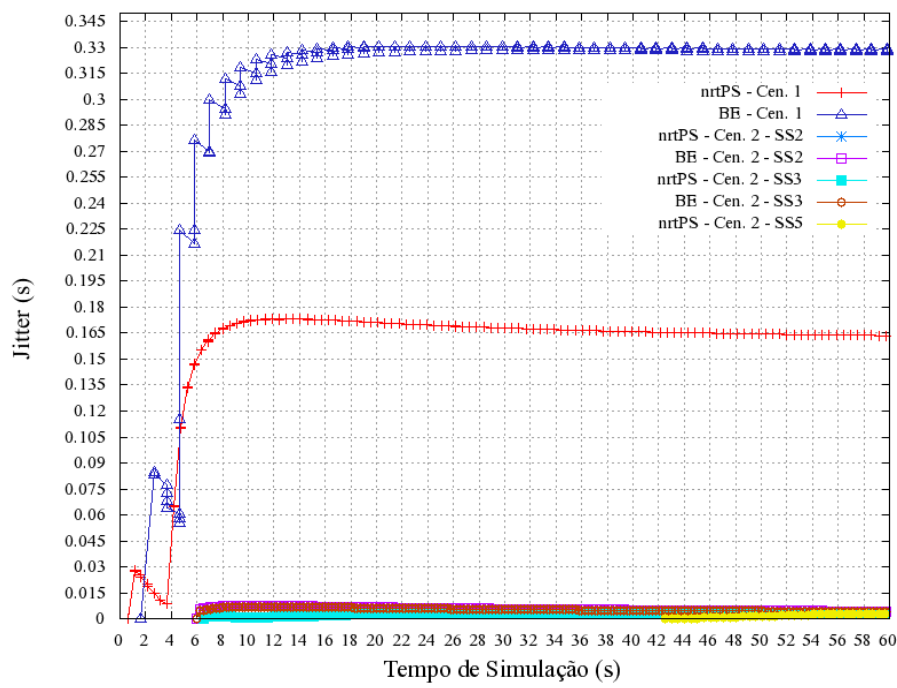

Figura 12: Jitter para tráfego TCP nrtPS e BE. revisada por Thomas Williams e Colin Kelley em 1986. Disponível em $<$ http://www.gnuplot.info >. Acesso em 15 de abril de 2007.

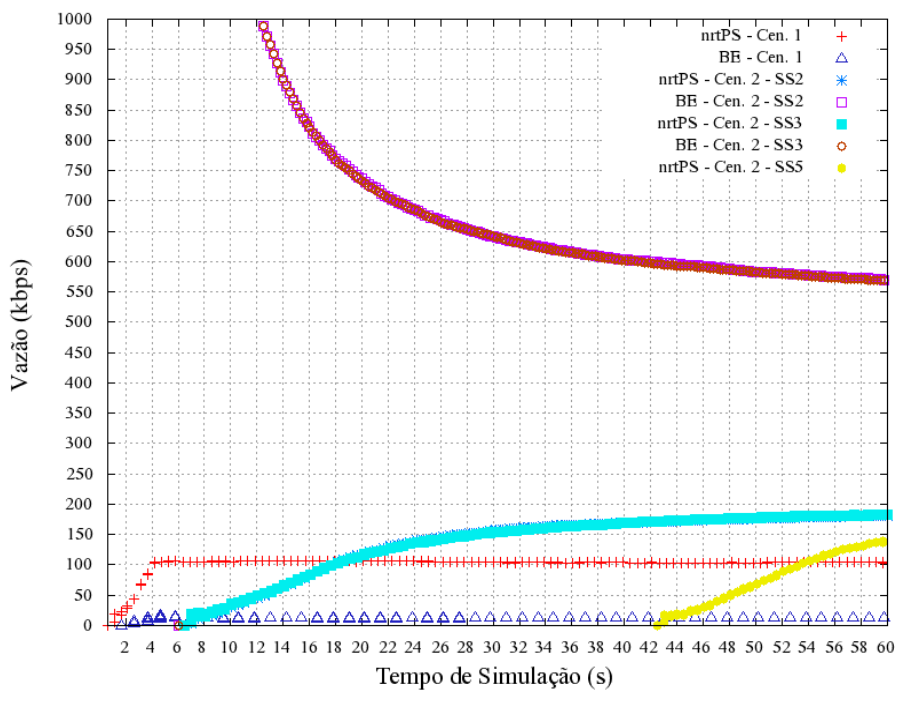

Figura 9: Vazão para os tráfegos TCP: nrtPS e BE.

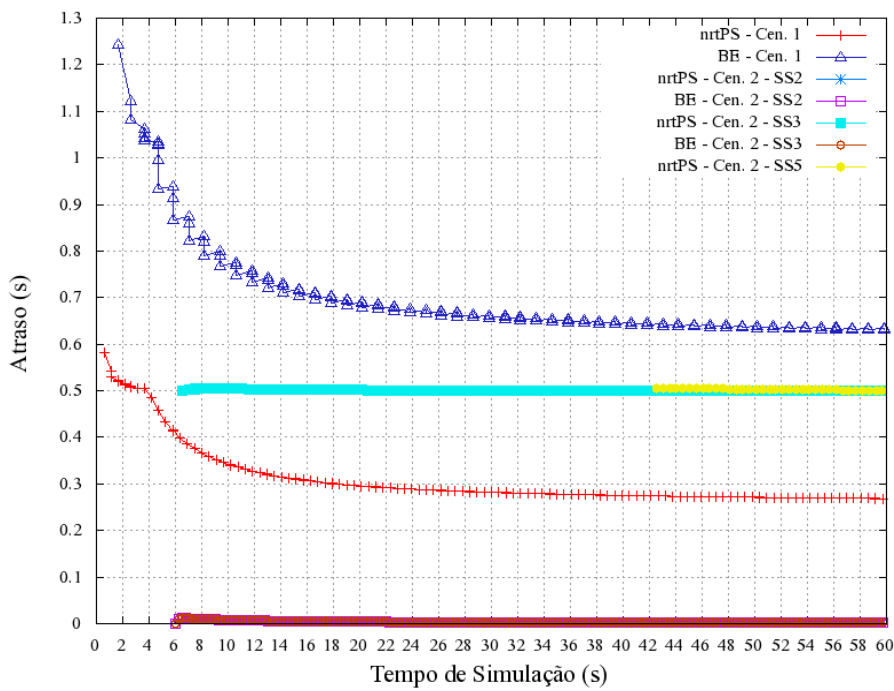

Figura 11: Atraso médio para tráfegos em TCP: nrtPS e BE.

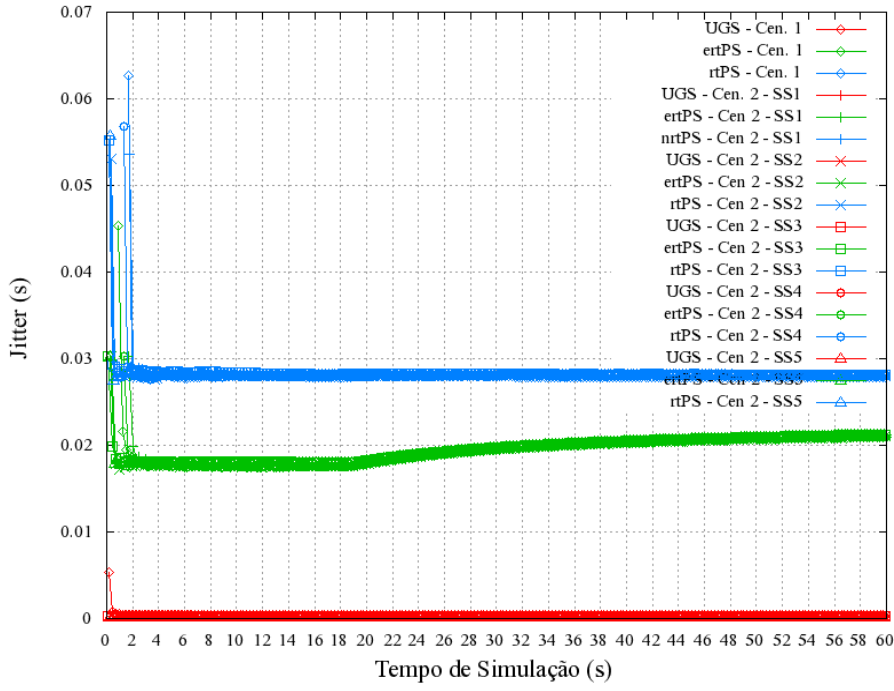

Figura 13: Jitter para tráfego UDP: UGS, ertPS e rtPS. 
Tabela 1: Parâmetros de simulação dos agentes geradores de tráfego

\begin{tabular}{lcccc} 
Tráfego & $\begin{array}{c}\text { Tx.mín de envio } \\
\text { (kbps) }\end{array}$ & $\begin{array}{c}\text { Tx.máx de envio } \\
\text { (kbps) }\end{array}$ & $\begin{array}{c}\text { Tamanho. dos pacotes } \\
\text { (bytes) }\end{array}$ & $\begin{array}{c}\text { Intervalo de envio } \\
\text { (s) }\end{array}$ \\
\hline UGS & 64 & 64 & 200 & $\mathrm{t}+(-0,5,0,5)^{*}$ \\
rtPS & 512 & 1024 & $(200,1000)^{*}$ & $\mathrm{t}+(-0,5,0,5)^{*}$ \\
ertPS & 8 & 64 & 200 & $\mathrm{t}+(-0,5,0,5)^{*}$ \\
nrtPS & 512 & 1024 & $(200,1000)^{*}$ & 0,01 \\
BE & - & 1024 & $(200,1000)^{*}$ & 0,01 \\
\hline
\end{tabular}

* distribuição uniforme e aleatória;

$\mathrm{t}$ = tempo em que o tráfego inicia.

\begin{tabular}{ll} 
Tabela 2: Camada MAC & \\
\hline Parâmetro & Valor \\
\hline Taxa DL/UL & $3: 2$ \\
Número de símbolos OFDMA/quadro & 49 \\
Número de subcanais & 30 \\
$\mathrm{CW}_{\min }$ & 32 opps \\
$\mathrm{CW}_{\max }$ & 1024 opps \\
\hline
\end{tabular}

\begin{tabular}{ll} 
Tabela 3: Camada Física & \\
\hline Parâmetro & Valor \\
\hline Freqüência de operação & $5,0 \mathrm{GHz}$ \\
Largura de banda (BW) & $20 \mathrm{MHz}$ \\
64 QAM $3 / 4$ & $-63 \mathrm{dBm}$ \\
\hline
\end{tabular}

Tabela 4: Média, Desvio-Padrão e Intervalos de Confiança de 95\% dos parâmetros médios de Qos da simulação do Cenário 1

\begin{tabular}{c|l|c|c|c|c}
\hline \multirow{2}{*}{ Parâmetro } & \multicolumn{2}{|c|}{ Tráfego } & \multirow{2}{*}{ Média } & \multirow{2}{*}{ Desvio-Padrão } & \multicolumn{2}{c}{ Intervalo de Confiança } \\
\cline { 5 - 6 } Atraso Médio & UGS & 0,000292572 & 0,000142495 & 0,000286864 & 0,000298281 \\
& ertPS & 0,019747178 & 0,001510510 & 0,019682832 & 0,019811523 \\
& rtPS & 0,028171770 & 0,000701516 & 0,028159446 & 0,028184093 \\
& nrtPS & 0,501003648 & 0,003443145 & 0,500862655 & 0,501144642 \\
& BE & 2,869762018 & 0,568245406 & 2,807206968 & 2,932317067 \\
\hline \multirow{5}{*}{ Jitter } & UGS & 0,000002207 & 0,000055015 & 0,000000003 & 0,000004411 \\
& ertPS & 0,000049442 & 0,000409654 & 0,000031991 & 0,000066893 \\
& rtPS & 0,000010138 & 0,000090996 & 0,000008539 & 0,000011736 \\
& nrtPS & 0,000055111 & 0,001313369 & 0,000001330 & 0,000108892 \\
& BE & 0,010364000 & 0,022348398 & 0,007903787 & 0,012824214 \\
\hline \multirow{5}{*}{ Vazão Média } & UGS & 64,222950613 & 1,660748446 & 64,156506837 & 64,289394389 \\
& ertPS & 58,030510399 & 2,652860973 & 57,918424900 & 58,142595897 \\
& rtPS & $1.026,532192244$ & 52,213121859 & $1.025,627363254$ & $1.027,437021235$ \\
& nrtPS & 194,787037161 & 18,819471026 & 194,019574078 & 195,554500243 \\
& BE & 31,041262354 & 3,980405730 & 30,615650441 & 31,466874267 \\
\hline
\end{tabular}

